REVISTA

\title{
CARACTERIZAÇÃO MICROESTRUTURAL E PROPRIEDADES FÍSICAS E MECẤNICAS DE ARGILAS DA REGIÃO NORTE DO ESPÍRITO SANTO
}

\author{
MICROSTRUCTURAL CHARACTERIZATION AND PHYSICAL \\ MECHANICAL PROPERTIES OF CLAYS FROM THE NORTHERN OF \\ ESPIRITO SANTO
}

\author{
${ }^{1}$ Júlia Moura Nascimento \\ ${ }^{2}$ Juliana Patrocinio Martins \\ ${ }^{3}$ Ana Paula Castello de Lima \\ ${ }^{4}$ Sara de Jesus Bulhosa \\ ${ }^{5}$ Vanessa Creto Bellato \\ ${ }^{6}$ Carla Therezinha Dalvi Borjaille Alledi \\ ${ }^{7}$ Georgia Serafim Araújo \\ ${ }^{8 *}$ André Gustavo de Sousa Galdino
}

\begin{abstract}
${ }^{1}$ Instituto Federal do Espírito Santo, Campus Vitória. E-mail: juliamoura_12@hotmail.com
${ }^{2}$ Instituto Federal do Espírito Santo, Campus Vitória. E-mail: juju.patrocinio@hotmail.com ${ }^{3}$ Instituto Federal do Espírito Santo, Campus Vitória. E-mail: anapaulacastellodelima@hotmail.com ${ }^{4}$ Instituto Federal do Espírito Santo, Campus Vitória. E-mail: sarabulhosa@hotmail.com ${ }^{5}$ Universidade Federal do Espírito Santo, Campus Goiabeiras. E-mail: vanessa_creto@hotmail.com ${ }^{6}$ Instituto Federal do Espírito Santo, Campus Vitória. E-mail: borjaille@ifes.edu.br ${ }^{7}$ Instituto Federal do Espírito Santo, Campus Vitória. E-mail: georgia@ifes.edu.br ${ }^{8}$ Instituto Federal do Espírito Santo, Campus Vitória. E-mail: andregsg@ifes.edu.br *Autor de correspondência
\end{abstract}

Artigo submetido em 13/02/2020, aceito em 04/05/2020 e publicado em 28/08/2020.

Resumo: O objetivo deste trabalho foi determinar as propriedades mineralógicas, físicas e mecânicas de argilas do norte do Espírito Santo. Foram analisadas duas argilas na forma como recebido (amostras A e B) e dois traços (2A-5B - amostra C; e 3A-4B - amostra D) utilizadas para confecção de tijolos. Para caracterização mineralógica utilizou-se as técnicas de fluorescência de raios $\mathrm{X}$, microscopia eletrônica de varredura, difração de raios $\mathrm{X}$, distribuição granulométrica, análises térmica diferencial e termogravimétrica e limites de Atterberg. Avaliou-se também absorção de água, porosidade aparente, massa específica aparente, retração linear após queima e limite de resistência à compressão de corpos de prova com $12 \mathrm{~mm}$ de diâmetro por $24 \mathrm{~mm}$ de altura, confeccionados por prensagem uniaxial, secados a $110^{\circ} \mathrm{C}$ por $24 \mathrm{~h}$ e sinterizados a $850^{\circ} \mathrm{C}, 900^{\circ} \mathrm{C}$ e $950^{\circ} \mathrm{C}$, a $5^{\circ} \mathrm{C} / \mathrm{min}$ com permanência por $2 \mathrm{~h}$ nessas temperaturas. Os resultados das propriedades mineralógicas das amostras A e B apresentaramse de acordo com a literatura. Os resultados das propriedades físicas e mecânicas apontaram para a utilização da amostra D como matéria prima para fabricação de tijolos. 
Palavras-chave: Argilas para cerâmica vermelha; propriedades mineralógicas; propriedades físicas e mecânicas.

\begin{abstract}
This work aimed to determine mineralogical, physical and mechanical properties of clays from the north of Espírito Santo. We analyzed two clays as received (samples A and B) and two traces (2A-5B - sample C; and 3A-4B - sample D). In order to determine the mineralogical properties of samples, we used $\mathrm{X}$ rays fluorescence, scanning electronic microscopy, $\mathrm{X}$ rays diffraction, granulometric analysis, differential thermal analysis, thermogravimetric analysis and Atterberg limits. . Also, we evaluated water absorption, apparent porosity, apparent specific gravity, linear retraction after firing and ultimate compressive strength using specimens with diameter of $12 \mathrm{~mm}$ and height of $24 \mathrm{~mm}$, made by uniaxial pressing, dried at $110^{\circ} \mathrm{C}$ for $24 \mathrm{~h}$ and fired at $850^{\circ} \mathrm{C}, 900^{\circ} \mathrm{C}$ and $950^{\circ} \mathrm{C}$, with $5^{\circ} \mathrm{C} / \mathrm{min}$ and staying at those temperatures for $2 \mathrm{~h}$. Mineralogical properties results for samples $\mathrm{A}$ and $\mathrm{B}$ were in accordance to literature. Physical and mechanical properties results pointed to the use of sample D as raw material to make bricks.
\end{abstract}

Keywords: Red clays; mineralogical properties; physical and mechanical properties.

\section{INTRODUÇÃO}

O Brasil é um país rico em jazidas de argilas, cujas aplicações têm sido principalmente para a indústria da cerâmica vermelha. Essa indústria é responsável pela fabricação de peças, como, por exemplo, tijolos, telhas, tubos e blocos cerâmicos para utilização na construção civil, e que, em 2011, a produção de 84,8 bilhões de peças no Brasil, com $70 \%$ dessa produção referente a blocos e tijolos e $30 \%$ a telhas (AGÊNCIA BRASILEIRA DE DESENVOLVIMENTO INDUSTRIAL, 2016). As argilas são de origem natural, terrosa, com granulometria fina que apresenta plasticidade se for umedecida com água e que são formados por argilominerais, tais como os silicatos hidratados de alumínio, ferro e magnésio. Uma vez que as argilas podem ser compostas por combinações de diversos argilominerais, elas podem ser classificadas em função da sua origem, composição química e concentração de argilominerais (BRITO; ALMEIDA; NEVES; MENEZES; SILVA; SANTANA, 2015).

Os argilominerais presentes na argila são responsáveis pelas características das argilas, a saber: desenvolvimento de plasticidade; elevada área superficial; solubilidade; e instabilidade térmica (VIEIRA; TERRONES; SÁNCHEZ; MONTEIRO, 2007). Entretanto, na extração das argilas, também há a presença de areia e silte, bem como de camadas de argilas de diferentes composições, o que pode causar alteração nas propriedades dos produtos fabricados. Além disso, ao longo da extração da jazida, as camadas de argila podem evoluir em suas propriedades com o tempo de consumo ou mesmo empreender a exploração em outras zonas de uma mesma mina (SANCHEZ; GARCÍA, GINÉS; NEGRE, 1996).

As argilas utilizadas para fabricar materiais de construção civil (telhas, tijolos de alvenaria e furados, ladrilhos de piso, etc.) precisam ser fáceis de moldar, ter valor médio ou elevado de módulo de ruptura à flexão (antes e após queima), e costumam ter cor avermelhada, após queima em baixas temperaturas (por volta de $900^{\circ} \mathrm{C}$ ), com mínimo de trincas e empenamentos. Porém, se essas argilas possuírem elevados teores de ferro bivalente, elementos alcalinos e alcalino-terrosos, os produtos fabricados podem apresentar retração excessiva, redução da faixa de vitrificação e colorações indesejáveis. Entretanto, nem todas as olarias utilizam argilas adequadas para fabricação dos materiais de construção civil, o que faz com que esses não tenham a melhor qualidade, além de não atenderem às normas ABNT, devido ao processo de fabricação. Vários autores têm reportado a análise dos resultados das propriedades tecnológicas e mineralógicas das argilas utilizadas na fabricação de artefatos da 
indústria da cerâmica vermelha (DE MORAIS; SPOSTO, 2006; VIEIRA; FEITOSA; MONTEIRO, 2003; CAMPOS et al., 1999; ROCHA BARRETO; DA COSTA, 2018, CARREIRO et al., 2016; MEDEIROS et al., 2017; RAMOS et al., 2010; TEIXEIRA; DE SOUZA; MOURA, 2001; VIEIRA; MONTEIRO; DUAILIBI FILHO, 2001; MACEDO et al., 2008; SAMUDIO PÉREZA et al., 2010; ALBERS et al., 2002), além do perfil das principais indústrias cerâmicas e seus produtos (MOTTA; ZANARDO; CABRAL JUNIOR, 2002) e dos aspectos sobre a extrusão de massas cerâmicas vermelhas (RIBEIRO; FERREIRA; LABRINCHA, 2003; SANTOS et al., 2012).

Este trabalho teve como objetivo avaliar as propriedades mineralógicas, físicas e mecânicas das argilas utilizadas para fabricação de tijolos e blocos estruturais da Cerâmica Adélio Lubiana Ltda., situada no norte do Espírito Santo.

\section{PROCESSOS METODOLÓGICOS: MATERIAIS E MÉTODOS}

\subsection{Materiais}

Foram coletadas duas argilas plásticas gentilmente doadas pela Cerâmica Adélio Lubiana Ltda., localizada no município de Nova Venécia, ES: amostra A e amostra B. Inicialmente, essas argilas foram peneiradas em peneira ABNT n. $80(0,177 \mathrm{~mm})$. As argilas foram misturadas em traços de duas partes da amostra A para cinco partes da amostra B (5B-2A - amostra C) e de três partes da amostra A para quatro partes da amostra B (4B-3A - amostra D), para reproduzir os traços utilizados pela empresa na fabricação de tijolos e blocos cerâmicos de alvenaria.

\subsection{Métodos}

As amostras A e B foram submetidas aos ensaios de limites de Atterberg (limites de plasticidade - LP e de liquidez - LL) e calculado o índice de plasticidade (IP); difração de raios $\mathrm{X}$ (DRX); microscopia eletrônica de varredura (MEV); análise termogravimétrica (DTA/TGA); e análise granulométrica.

Para os ensaios físicos de absorção de água (AA), porosidade aparente (PA), cor após queima (COR), retração linear de queima (RLQ), massa específica aparente (MEA) e para o ensaio mecânico de limite de resistência à compressão (LRC), foram confeccionados corpos de prova cilíndricos com dimensões de $12 \mathrm{~mm}$ de diâmetro por $24 \mathrm{~mm}$ de altura, por meio de prensagem uniaxial. Esses corpos de prova foram secados a $110^{\circ} \mathrm{C}$ por $24 \mathrm{~h}$ e, em seguida, sinterizados a $850^{\circ} \mathrm{C}, 900^{\circ} \mathrm{C}$ e $950^{\circ} \mathrm{C}$, com rampa de aquecimento de $5^{\circ} \mathrm{C} / \mathrm{min}$ e tempo de permanência de $2 \mathrm{~h}$. A metodologia utilizada foi adaptada da proposta por Souza Santos (1989).

Para o cálculo da absorção de água (AA), massa específica aparente (MEA) e porosidade aparente (PA), foram utilizadas as Equações (A), (B) e (C), respectivamente.

$$
\begin{aligned}
& A A(\%)=\left(\frac{P_{U}-P_{S}}{P_{S}}\right) \cdot 100(\mathrm{~A}) \\
& M E A\left(g / \mathrm{cm}^{3}\right)=\left(\frac{P_{S}}{P_{U}-P_{l}}\right)(\mathrm{B}) \\
& P A(\%)=\left(\frac{P_{U}-P_{S}}{P_{U}-P_{l}}\right) \cdot 100(\mathrm{C})
\end{aligned}
$$

onde $\mathrm{P}_{\mathrm{I}}$ é o peso imerso, $\mathrm{P}_{\mathrm{S}}$ é o peso seco e $\mathrm{P}_{\mathrm{U}}$ é o peso úmido.

A retração linear de queima foi calculada com base na Equação (D) abaixo.

$$
R L Q(\%)=\frac{L_{1}-L_{2}}{L_{z}} \cdot 100(\mathrm{D})
$$

onde $L_{l}$ é a altura inicial do corpo de prova, antes da queima, e $L_{2}$ a altura final do corpo de prova, após queima.

Para o limite de resistência à compressão dos corpos de prova se utilizou a equação (E). 


$$
T(M P a)=\frac{F}{s}(\mathrm{E})
$$

onde $\mathrm{F}$ é a carga de compressão, $\mathrm{S}$ é a área da seção transversal do corpo de prova e $\mathrm{T}$ é o limite de resistência à compressão.

Todos os resultados foram obtidos da média aritmética e desvio padrão de cinco corpos de prova por temperatura de queima.

Essas temperaturas foram escolhidas por serem as usualmente utilizadas pelas empresas de cerâmica vermelha para queima de seus produtos. Os ensaios físicos (AA, PA, MEA) foram realizados por meio do princípio de Arquimedes, enquanto o ensaio de compressão foi realizado em uma máquina universal de ensaios Emic, calibrada para $70.000 \mathrm{~N}$.

\section{RESULTADOS E DISCUSSÃO}

Primeiramente, foi realizada análise química das duas argilas através de Fluorescência de Raios X (FRX), para determinação da composição química das duas argilas. As duas argilas apresentam características diferentes, onde uma é mais plástica que a outra. A argila mais plástica será chamada de argila $\mathrm{A}$, enquanto a menos plástica de argila B. A Tabela 1 ilustra a composição química das duas argilas.

Observa-se a presença dos três principais compostos de argilas que são utilizados para a indústria de cerâmica vermelha, a saber: $\mathrm{SiO}_{2}, \mathrm{Al}_{2} \mathrm{O}_{3}$ e $\mathrm{Fe}_{2} \mathrm{O}_{3}$. Esses três compostos são responsáveis por $96,269 \%$ da composição química da argila A, e por $95,189 \%$ da argila B.

De acordo com Durães (1983), as argilas para fabricação de tijolos devem ter as seguintes características:

- plasticidade - as argilas plásticas são aquelas que, adicionadas de quantidade adequada de água, passam ao estado pastoso e se deixam moldar facilmente por extrusão, conservando a forma moldada até a secagem, quando se tornam mecanicamente duras e bastante resistentes. A pasta moldável não deve apresentar-se arenosa ao tato;

Tabela 1: Resultados das análises químicas das amostras A e B

\begin{tabular}{|c|c|c|}
\hline \multirow{2}{*}{ Composto } & \multicolumn{2}{|c|}{ Composição química (\%) } \\
\hline & Argila A & Argila B \\
\hline $\mathrm{SiO}_{2}$ & 47,353 & 51,974 \\
\hline $\mathrm{Al}_{2} \mathrm{O}_{3}$ & 35,598 & 35,711 \\
\hline $\mathrm{Fe}_{2} \mathrm{O}_{3}$ & 13,318 & 7,504 \\
\hline $\mathrm{K}_{2} \mathrm{O}$ & 1,268 & 2,305 \\
\hline $\mathrm{TiO}_{2}$ & 0,909 & 0,987 \\
\hline $\mathrm{MgO}$ & 0,823 & 1,297 \\
\hline $\mathrm{SO}_{3}$ & 0,404 & $\mathrm{n} / \mathrm{d}$ \\
\hline $\mathrm{BaO}$ & 0,309 & $\mathrm{n} / \mathrm{d}$ \\
\hline $\mathrm{ZnO}$ & 0,018 & $\mathrm{n} / \mathrm{d}$ \\
\hline $\mathrm{V}_{2} \mathrm{O}_{5}$ & $\mathrm{n} / \mathrm{d}$ & 0,068 \\
\hline $\mathrm{MnO}$ & $\mathrm{n} / \mathrm{d}$ & 0,053 \\
\hline $\mathrm{ZrO}_{2}$ & $\mathrm{n} / \mathrm{d}$ & 0,052 \\
\hline $\mathrm{CuO}$ & $\mathrm{n} / \mathrm{d}$ & 0,030 \\
\hline $\mathrm{Rb}_{2} \mathrm{O}$ & $\mathrm{n} / \mathrm{d}$ & 0,019 \\
\hline
\end{tabular}

n/d - não determinado

Fonte: Autoria própria (2016).

- ferruginosas - são as que contêm uma porcentagem de até $5 \%$ de óxidos ou hidróxidos de ferro. Os óxidos de ferro, até o limite máximo de $5 \%$, são benéficos, dando aos tijolos a coloração avermelhada desejada, além de facilitar a ceramização durante a queima;

- fundentes - são argilas contendo impurezas de metais alcalinos, que lhes fornecem propriedades de sinterização à baixa temperatura. A presença dessas impurezas na argila permite que os tijolos sejam queimados a temperaturas mais baixas com economia de combustíveis e tempo de queima;e

- silicosas ou argilas magras - são as que contêm alto teor de anidrido silícico $\left(\mathrm{SiO}_{2}\right)$. São utilizadas para reduzir a plasticidade excessiva das argilas muito plásticas. Entretanto, o excesso de argila silicosa enfraquece o tijolo, aumentando sua porosidade após a queima (DURÃES, 1983). 
Observa-se, assim, que a argila A possui impurezas de metais alcalinos e alcalinos-terrosos $(\mathrm{K}, \mathrm{Mg}, \mathrm{Ba})$, bem como a argila $\mathrm{B}(\mathrm{K}, \mathrm{Mg})$. Para as duas argilas, o teor de $\mathrm{Fe}_{2} \mathrm{O}_{3}$ é superior a $5 \%$. Também se observa valores razoáveis de $\mathrm{SiO}_{2}$.

Os resultados dos ensaios, para obtenção dos limites de Atterberg e do índice de plasticidade para as amostras $\mathrm{A}$ e B, estão ilustrados na Tabela 2.

Observa-se que as duas amostras são muito plásticas (IP $>15 \%$ ), sendo a amostra A mais plástica que a $\mathrm{B}$. De acordo com Santos et al. (2012), para que se tenha uma extrusão aceitável, a amostra deve apresentar índice de plasticidade entre $10 \mathrm{e}$ $34 \%$ e limite de plasticidade entre 18 e $31 \%$, enquanto para uma extrusão ótima, esses valores devem ser de 15 a $25 \%$ para o IP e de 18 a $25 \%$ para LP.

Tabela 2: Resultados de limites de Atterberg e índice de plasticidade das amostras A e B

\begin{tabular}{ccc}
\hline \multirow{2}{*}{ CARACTERÍSTICA } & \multicolumn{2}{c}{ AMOSTRA } \\
\cline { 2 - 3 } & $\mathrm{A}$ & $\mathrm{B}$ \\
\hline $\begin{array}{c}\text { Limite de liquidez (LL) } \\
\text { [\%] }\end{array}$ & 80 & 55 \\
$\begin{array}{c}\text { Limite de plasticidade } \\
\text { (LP) [\%] }\end{array}$ & 44 & 38 \\
$\begin{array}{c}\text { Índice de plasticidade } \\
\text { (IP) [\%] }\end{array}$ & 36 & 17 \\
\hline
\end{tabular}

Fonte: Autoria própria (2016).

Dessa forma, observa-se que para o índice de plasticidade, a amostra B apresenta IP para extrusão ótima, embora o valor de LP presente seja superior ao sugerido para extrusão aceitável. A amostra A apresenta valores superiores de IP e LP aos definidos pelos autores. Isso explica o porquê de a empresa trabalhar com traços 3A-4B e 2A-5B para fabricação de produtos cerâmicos (tijolos e blocos cerâmicos).

A Figura 1 ilustra os resultados de difração de raios $\mathrm{X}$ (DRX) para as amostras A e B. É possível observar os picos principais da caulinita e da ilita para as duas amostras. As argilas plásticas empregadas para cerâmica vermelha ou estrutural possuem composição mineralógica composta por caulinita com ilita $\left(\mathrm{K}_{\mathrm{x}}(\mathrm{Al}\right.$, $\mathrm{Mg})_{4}(\mathrm{Si}, \mathrm{Al})_{8} \mathrm{O}_{20}(\mathrm{OH})_{4} \cdot n \mathrm{H}_{2} \mathrm{O}$, com $\left.\mathrm{x}<1\right)$ ou montmorilonita ( $(\mathrm{Mg}, \mathrm{Ca}) \mathrm{O}$. $\left.\mathrm{Al}_{2} \mathrm{O}_{3} \mathrm{Si}_{5} \mathrm{O}_{10} . n \mathrm{H}_{2} \mathrm{O}\right)$ ou com esses minerais em camadas mistas, além de teor apreciável de ferro na forma de hidróxidos férricos (SOUZA SANTOS, 1989). Para as amostras em questão, não foi detectada montmorilonita. A ficha utilizada para a caulinita foi a PDF 01-080-0886 e para a ilita foi a PDF 00-026-0911.

As micrografias das argilas A e B obtidas por microscopia eletrônica de varredura (MEV) estão ilustradas na Figura 2.

Verificou-se que as partículas encontram-se agregadas, o que caracteriza que essa amostra é bastante plástica. Isso é corroborado com o índice de plasticidade da amostra A igual a $36 \%$, descrito na Tabela 2. Para a amostra $B$, é possível verificar na Figura 2(b) que as partículas também estão agregadas, porém com tamanho agregado inferior aos da amostra A, o que caracteriza que a amostra $\mathrm{B}$ é menos plástica que a $\mathrm{A}$, como indicado pelo índice de plasticidade da amostra B, que é igual a $17 \%$. 
Figura 1: Difratograma de raios $\mathrm{X}$ das amostras: (a) A; e (b) B

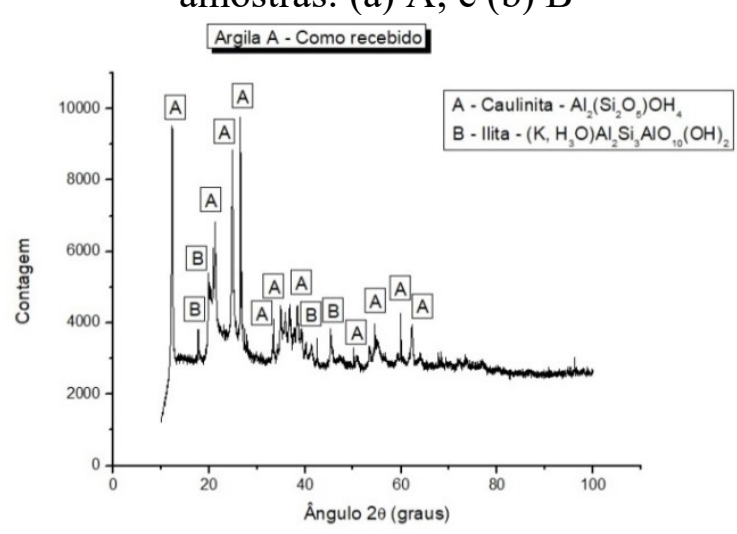

(a)

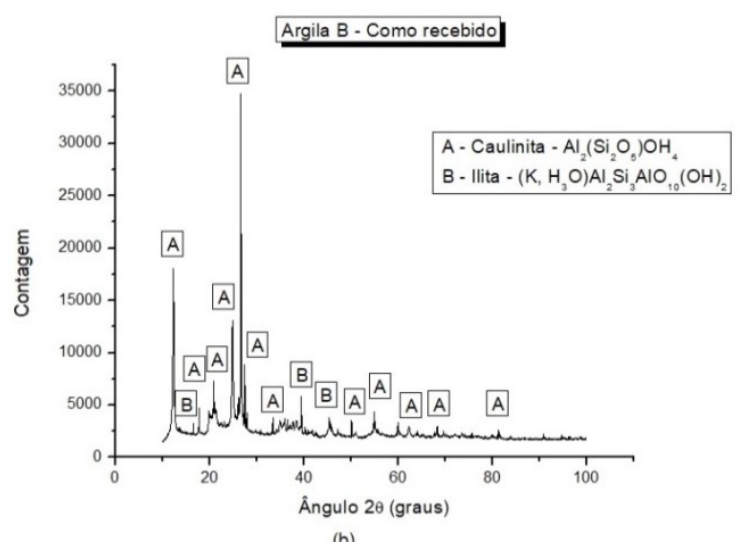

Fonte: Autoria própria (2016)

Os resultados da análise granulométrica das amostras A e B estão representados na Figura 3.

Observa-se que as partículas para a amostra A variaram de 0,363 $\mu \mathrm{m}$ a 120,226 $\mu \mathrm{m}$, com valores de $\mathrm{d}(0,1)=3,229 \mu \mathrm{m}$, $\mathrm{d}(0,5)=23,289 \mu \mathrm{m}$ e $\mathrm{d}(0,9)=66,915 \mu \mathrm{m}$, ou seja, $90 \%$ das partículas possuem tamanho máximo de $66,915 \mu \mathrm{m}$. Já para a amostra B, as partículas variaram de 0,363 $\mu \mathrm{m}$ a $363,078 \mu \mathrm{m}$, com valores de $\mathrm{d}(0,1)=$ $3,169 \mu \mathrm{m}, \mathrm{d}(0,5)=22,685 \mu \mathrm{m}$ e $\mathrm{d}(0,9)=$ $97,875 \mu \mathrm{m}$, ou seja, $90 \%$ das partículas possuem tamanho máximo de $97,875 \mu \mathrm{m}$.
Figura 2: Micrografias das amostras: (a) A; e (b) B

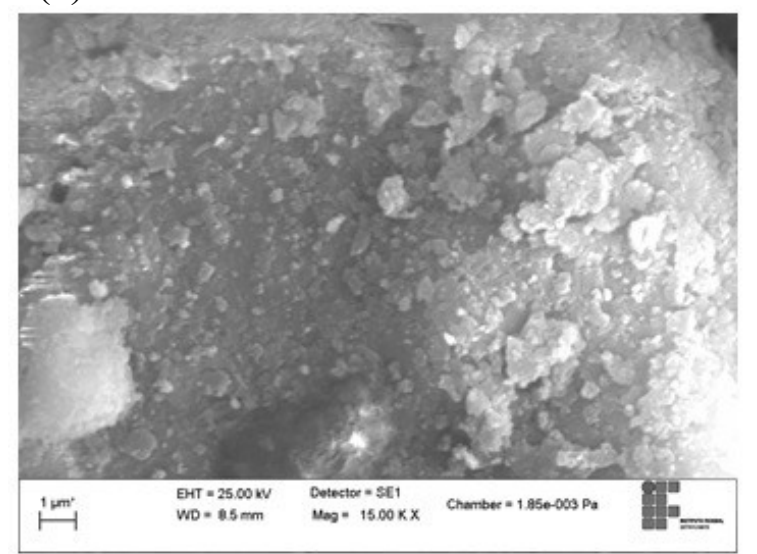

(a)

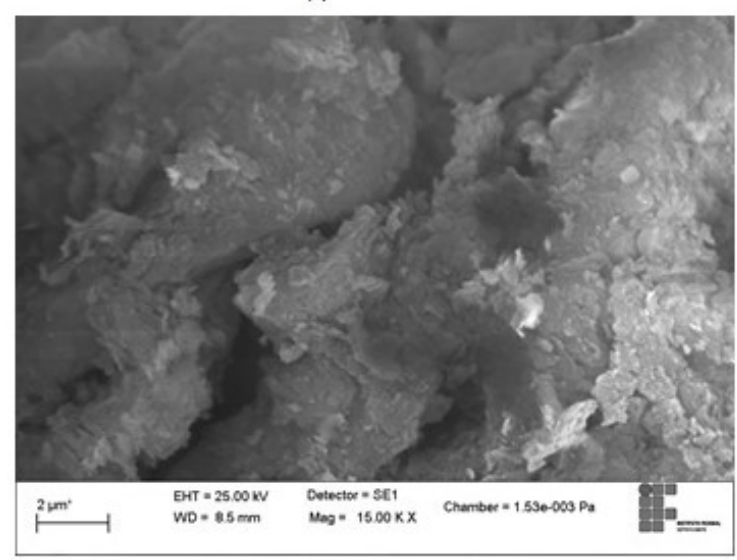

(b)

Fonte: Autoria própria (2016).

Observou-se também a diferença na distribuição de tamanho de partículas para as duas argilas, onde a amostra B apresenta distribuição mais uniforme que a $\mathrm{A}$. Em geral, as argilas utilizadas para fabricação de produtos de cerâmica vermelha ou estrutural possuem granulometria fina com teor apreciável de matéria orgânica, que são fatores responsáveis pela alta plasticidade das mesmas (SOUZA SANTOS, 1989).

Os resultados das análises térmicas diferenciais e termogravimétricas das argilas A e B estão ilustrados na Figura 4. 
Figura 3: Análise granulométrica das amostras: (a) A; e (b) B Distribuição de tamanho de particula

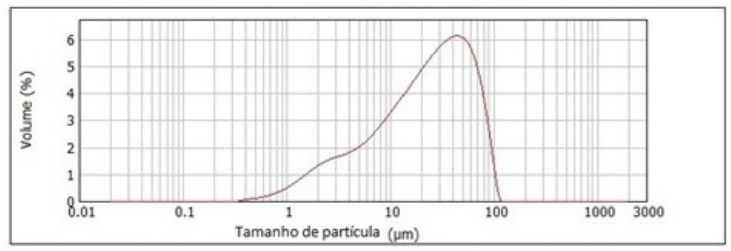

(a)

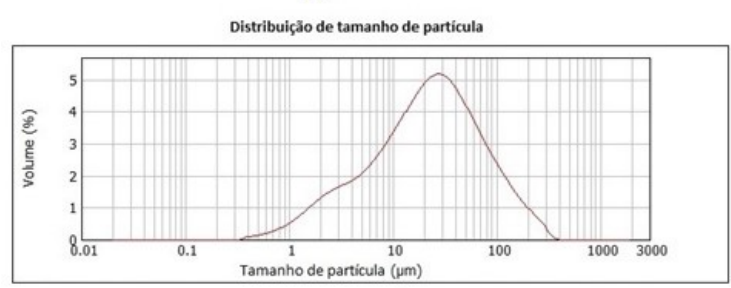

(b)

Fonte: Autoria própria (2016).

Para a amostra A, houve combustão de matéria orgânica a $327^{\circ} \mathrm{C}$, perda de hidroxila a $517^{\circ} \mathrm{C}$ e pico exotérmico a $958^{\circ} \mathrm{C}$ possivelmente relacionado à formação de espinélio, após a total destruição da estrutura da ilita, além de perda de massa de 15,83\%. Para a amostra $\mathrm{B}$, a combustão de matéria orgânica ocorreu a $307^{\circ} \mathrm{C}$, com a perda de hidroxila a $510^{\circ} \mathrm{C}$ e $960^{\circ} \mathrm{C}$, além de perda de massa de $12,46 \%$.

A Tabela 3 apresenta os resultados de massa específica aparente, porosidade aparente, absorção de água, retração linear de queima e cor para as amostras A, B, C e $\mathrm{D}$, após queima a $850^{\circ} \mathrm{C}, 900^{\circ} \mathrm{C}$ e $950^{\circ} \mathrm{C}$. Comparando-se os resultados obtidos na Tabela 3, com os valores especificados nas Tabelas 4 (BARZAGHI; SALGE, 1982) e 5 (SOUZA; SOUZA SANTOS, 1964), tem-se que:

Absorção de água - para a absorção de água, os valores variaram de $26,97 \pm 12,27 \%$ $\left(\right.$ amostra D $\left.-900^{\circ} \mathrm{C}\right)$ a $39,07 \pm 2,17 \%$ (amostra $\left.\mathrm{A}-850^{\circ} \mathrm{C}\right)$; apenas a amostra $\mathrm{D}$ com temperatura de queima a $900^{\circ} \mathrm{C}$ atendeu à especificação, que é de no máximo $25 \%$.
Figura 4: Análise térmica diferencial e termogravimétrica das amostras: (a) A; e (b) B

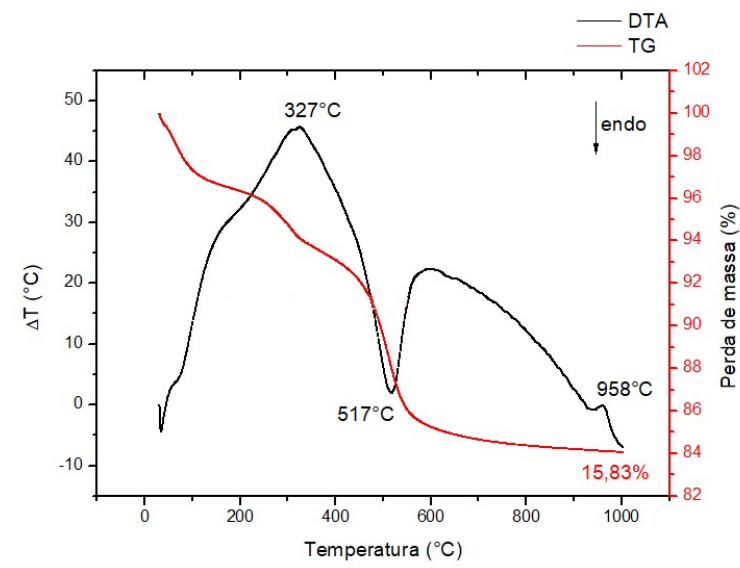

(a)

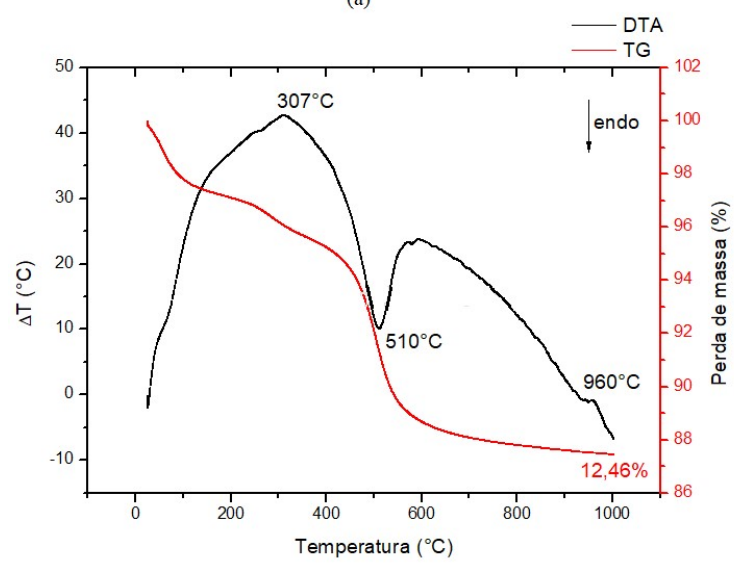

(b)

Fonte: Autoria própria (2016).

massa especifica aparente - para massa específica aparente, tem-se que os valores variaram de $1,64 \pm 0,04 \mathrm{~g} / \mathrm{cm}^{3}$ (amostra $\mathrm{A}$ $850^{\circ} \mathrm{C}$ ) a $1,80 \pm 0,07 \mathrm{~g} / \mathrm{cm}^{3}$ (amostra C $850^{\circ} \mathrm{C}$ ); apenas as amostras $\mathrm{C}$ e $\mathrm{D}$ atenderam à especificação nas três temperaturas de queima, que é de no mínimo $1,70 \mathrm{~g} / \mathrm{cm}^{3}$.

Porosidade aparente - para porosidade aparente, tem-se que os valores variaram de $28,78 \pm 12,83 \% \quad\left(\right.$ amostra $\left.\mathrm{D}-900^{\circ} \mathrm{C}\right)$ a $41,02 \pm 0,87 \%$ (amostra $\mathrm{A}-850^{\circ} \mathrm{C}$ ); apenas a amostra $\mathrm{D}$ atendeu à especificação nas temperaturas de $900^{\circ} \mathrm{C}$ e $950^{\circ} \mathrm{C}$, que é de no máximo 35\%.

Retração linear de queima - para retração linear de queima, tem-se que os valores variaram de $0,64 \pm 0,24 \%$ (amostra B - 
$\left.850^{\circ} \mathrm{C}\right)$ a $3,92 \pm 0,66 \%\left(\operatorname{amostra} \mathrm{A}-950^{\circ} \mathrm{C}\right)$; para este ensaio não há especificação.

$\underline{\mathrm{Cor}}$ - as cores obtidas após queima variaram de vermelho claro a vermelho escuro; esse ensaio, associado aos outros, sugere que para colorações avermelhadas ou um pouco mais claras ou escuras podem ser utilizadas na indústria de cerâmica vermelha.

A Tabela 6 ilustra os resultados do limite de resistência à compressão das amostras A, B, C e D, após sinterização a $850^{\circ} \mathrm{C}, 900^{\circ} \mathrm{C}$ e $950^{\circ} \mathrm{C}$.

Tabela 3: Resultados de propriedades físicas das amostras A, B, C e D

\begin{tabular}{|c|c|c|c|c|c|}
\hline \multirow{2}{*}{$\begin{array}{l}\text { Temperatura } \\
\text { de sinterização }\end{array}$} & \multirow{2}{*}{ Propriedade } & \multicolumn{4}{|c|}{ Amostra } \\
\hline & & $\mathrm{A}$ & $\mathrm{B}$ & $\mathrm{C}$ & $\mathrm{D}$ \\
\hline \multirow{5}{*}{$850^{\circ} \mathrm{C}$} & $\operatorname{MEA}\left(\mathrm{g} / \mathrm{cm}^{3}\right)$ & $1,64 \pm 0,04$ & $1,68 \pm 0,03$ & $1,80 \pm 0,07$ & $1,68 \pm 0,04$ \\
\hline & PA (\%) & $40,87 \pm 1,39$ & $37,92 \pm 0,89$ & $34,85 \pm 5,19$ & $37,13 \pm 4,01$ \\
\hline & AA (\%) & $39,07 \pm 2,17$ & $35,94 \pm 1,45$ & $30,32 \pm 4,85$ & $35,20 \pm 3,78$ \\
\hline & RLQ (\%) & $2,96 \pm 0,83$ & $0,64 \pm 0,24$ & $1,92 \pm 0,35$ & $1,32 \pm 0,58$ \\
\hline & COR & $\begin{array}{l}\text { Vermelho } \\
\text { escuro }\end{array}$ & $\begin{array}{l}\text { Laranja } \\
\text { claro }\end{array}$ & $\begin{array}{l}\text { Vermelho } \\
\text { claro }\end{array}$ & $\begin{array}{l}\text { Laranja } \\
\text { escuro }\end{array}$ \\
\hline \multirow{5}{*}{$900^{\circ} \mathrm{C}$} & $\operatorname{MEA}\left(\mathrm{g} / \mathrm{cm}^{3}\right)$ & $1,65 \pm 0,03$ & $1,66 \pm 0,02$ & $1,70 \pm 0,11$ & $1,78 \pm 0,14$ \\
\hline & PA (\%) & $41,02 \pm 0,87$ & $39,36 \pm 0,63$ & $45,12 \pm 11,24$ & $28,78 \pm 12,83$ \\
\hline & AA $(\%)$ & $38,77 \pm 1,47$ & $37,25 \pm 1,12$ & $39,02 \pm 9,50$ & $26,97 \pm 12,27$ \\
\hline & RLQ (\%) & $3,68 \pm 0,32$ & $0,91 \pm 0,35$ & $2,69 \pm 0,26$ & $1,77 \pm 0,63$ \\
\hline & COR & $\begin{array}{l}\text { Vermelho } \\
\text { escuro }\end{array}$ & $\begin{array}{l}\text { Laranja } \\
\text { claro }\end{array}$ & $\begin{array}{l}\text { Vermelho } \\
\text { claro }\end{array}$ & $\begin{array}{l}\text { Laranja } \\
\text { escuro }\end{array}$ \\
\hline \multirow{5}{*}{$950^{\circ} \mathrm{C}$} & $\operatorname{MEA}\left(\mathrm{g} / \mathrm{cm}^{3}\right)$ & $1,68 \pm 0,01$ & $1,68 \pm 0,03$ & $1,76 \pm 0,10$ & $1,73 \pm 0,09$ \\
\hline & PA (\%) & $40,68 \pm 0,69$ & $39,20 \pm 1,06$ & $45,12 \pm 11,24$ & $33,08 \pm 8,92$ \\
\hline & $\mathrm{AA}(\%)$ & $37,33 \pm 0,87$ & $36,59 \pm 1,55$ & $35,34 \pm 8,29$ & $31,16 \pm 8,38$ \\
\hline & RLQ (\%) & $3,92 \pm 0,66$ & $1,16 \pm 0,49$ & $2,89 \pm 0,50$ & $2,88 \pm 0,51$ \\
\hline & COR & $\begin{array}{l}\text { Vermelho } \\
\text { escuro }\end{array}$ & $\begin{array}{l}\text { Laranja } \\
\text { claro }\end{array}$ & $\begin{array}{l}\text { Vermelho } \\
\text { claro }\end{array}$ & $\begin{array}{c}\text { Laranja } \\
\text { claro }\end{array}$ \\
\hline
\end{tabular}

Fonte: Autoria própria (2016).

Tabela 4: Especificações de argilas quanto ao uso.

\begin{tabular}{cc}
\hline Provável uso & Absorção de água (AA) \\
\hline Tijolos maciços & $20 \%$ \\
\hline Tijolos furados & $20 \%$ \\
\hline Telhas & $20 \%$ \\
\hline
\end{tabular}

Fonte: Adaptado de: Barzaghi e Salge (1982).

Tabela 5: Especificações para corpos de prova cerâmicos queimados à temperatura de $950^{\circ} \mathrm{C}$.

\begin{tabular}{cccccc}
\hline Propriedades & AA (\%) & $\begin{array}{c}\text { MEA } \\
\left(\mathrm{g} / \mathrm{cm}^{3}\right)\end{array}$ & PA (\%) & RLQ (\%) & COR \\
\hline Especificações & $<25$ & $\geq 1,70$ & $\leq 35$ & NE & NE \\
\hline
\end{tabular}

NE - Não especificado.

Fonte: Adaptado de: Souza e Souza Santos (1964). 
Tabela 6: Resultados do limite de resistência à compressão das amostras A, B, C e D

\begin{tabular}{cccccc}
\hline \multirow{2}{*}{ Temperatura } & \multirow{2}{*}{ Propriedade } & \multicolumn{4}{c}{ Amostra } \\
\cline { 3 - 6 } & & $\mathrm{A}$ & $\mathrm{B}$ & $\mathrm{C}$ & $\mathrm{D}$ \\
\hline $850^{\circ} \mathrm{C}$ & LRC $(\mathrm{MPa})$ & $23,72 \pm 4,28$ & $18,27 \pm 2,05$ & $23,99 \pm 3,08$ & $32,19 \pm 6,55$ \\
$900^{\circ} \mathrm{C}$ & LRC $(\mathrm{MPa})$ & $20,93 \pm 5,97$ & $26,92 \pm 2,77$ & $23,53 \pm 1,74$ & $42,30 \pm 11,43$ \\
$950^{\circ} \mathrm{C}$ & LRC $(\mathrm{MPa})$ & $24,34 \pm 6,50$ & $17,62 \pm 3,23$ & $22,16 \pm 4,68$ & $46,59 \pm 6,52$ \\
\hline
\end{tabular}

Fonte: Autoria própria (2016).

Tensão de ruptura à compressão - para tensão de ruptura à compressão, tem-se que os valores variaram de $17,62 \pm 3,23 \mathrm{MPa}$ (amostra B $-950^{\circ} \mathrm{C}$ ) a $46,59 \pm 6,52 \mathrm{MPa}$ (amostra $\mathrm{D}-950^{\circ} \mathrm{C}$ ); todas as amostras atenderam à especificação, que é de no mínimo 5,88 MPa.

Comparando os resultados de resistência à compressão com a norma ABNT NBR 15270-1 (ABNT, 2017), observa-se que os valores encontrados de limite de resistência à compressão das amostras utilizadas neste trabalho apresentaram valores bem superiores aos previstos pelas normas (limite de resistência à compressão maior ou igual a $1,5 \mathrm{MPa}$ para blocos usados com furos na horizontal e maior ou igual a $3 \mathrm{MPa}$ para blocos usados com furos na vertical) em todas as condições de temperatura de queima.

\section{CONCLUSÕES}

De acordo com os resultados de caracterização microestrutural, pode-se concluir que as amostras em questão possuem as características principais desejadas para as amostras que são utilizadas para a indústria de cerâmica vermelha, mais especificamente para a fabricação de tijolos, que é o produto principal fabricado pela Cerâmica Adélio Lubiana Ltda., empresa parceira para a realização deste trabalho.

Após análise de absorção de água, porosidade aparente, massa específica aparente, cor após queima, limite de resistência à compressão, limites de Atterberg e índice de plasticidade das amostras A, B, C e D, antes e após sinterização a $850^{\circ} \mathrm{C}, 900^{\circ} \mathrm{C}$ e $950^{\circ} \mathrm{C}$, podese concluir que:

a) apenas a amostra D atendeu aos requisitos de absorção de água e porosidade aparente estabelecidas por Barzaghi e Salge (1982) e Souza e Souza Santos (1964); e

b) as amostras $\mathrm{C}$ e $\mathrm{D}$ atenderam ao requisito de massa específica aparente estabelecida por Souza e Souza Santos (1964).

Todas as amostras atenderam ao requisito de resistência à compressão mínima estabelecida pela norma ABNT NBR 15270-1 (ABNT, 2017).

\section{AGRADECIMENTOS}

Ao CNPq, pelo suporte financeiro (Processo n. 469212/2014-4), à Cerâmica Adélio Lubiana Ltda., pelo fornecimento das matérias-primas utilizadas neste trabalho e ao Ifes, pelo suporte financeiro, via bolsas de iniciação científica júnior.

\section{REFERÊNCIAS}

Agência Brasileira de Desenvolvimento Industrial. Estudo técnico setorial da cerâmica vermelha. República Federativa do Brasil, 2016, 16. Disponível em: https://conhecimento.abdi.com.br/conhecim ento/Publicaes 1/Estudo\%20T\%C3\%A9cnic o\%20Setorial $\% 20 \mathrm{da} \% 20 \mathrm{Cer} \% \mathrm{C} 3 \% \mathrm{~A} 2 \mathrm{mica}$ $\% 20$ Vermelha $\% 20 \mathrm{p} \% \mathrm{C} 3 \%$ Al gina $\% 20 \% \mathrm{C} 3$ $\%$ BAnica $\% 20$ Cer $\%$ C3\%A2mica\%20Verme lha.pdf. Acesso em: 21 jan. 2019.

Associação Brasileira de Normas Técnicas. NBR 15270-1: Componentes cerâmicos - 
Blocos e tijolos para alvenaria Parte 1: Requisitos, 2017, pp. 1-26.

ALBERS, A. P. F.; MELCHIADES, F. G.; MACHADO, R.; BALDO, J. B.; BOSCHI, A. O. Um método simples de caracterização de argilominerais por difração de raios $\mathrm{X}$.

Cerâmica, São Paulo, v. 48 pp. 34-37, 2002.

Disponível em: http://dx.doi.org/

10.1590/S0366-69132002000100008.

Acesso em:

BARZAGHI, L.; SALGE, A. Argilas para materiais de Construção. Cerâmica, São Paulo, v. 28, n. 151, 1982, pp. 289-293.

BRITO, I. P.; ALMEIDA; E. P.; NEVES, G. A.; MENEZES, R. R.; SILVA, V. J.; SANTANA, L. N. L. Avaliação de novos depósitos de argilas do Estado da Paraíba visando sua aplicação como matériasprimas cerâmicas. Cerâmica, São Paulo, v. 61, 2015, pp. 391-398. Disponível em: http:// dx.doi.org/10.1590/0366-

6913201561360 1926. Acesso em:

CAMPOS, L. F. A.; DE MACEDO, R. S.; KIYOHARA, P. K.; FERREIRA, H. C. Características de plasticidade de argilas para uso em cerâmica vermelha ou estrutural. Cerâmica, São Paulo, v. 45, 1999, pp. 140-145. Disponível em: http:// dx.doi.org/10.1590/S0366-691319990005 00006. Acesso em:

CARREIRO, M. E. A.; SANTOS, R. C.; SILVA, V. J.; LIRA, H. L.; NEVES, G. A.; MENEZES, R. R.; SANTANA, L. N. L. Resíduo de quartzito - matéria-prima alternativa para uso em massas de cerâmica estrutural. Cerâmica, v. 62, 2016, pp. 170178. Disponível em: http://dx.doi.org/10. 1590/0366-691320 16623621990. Acesso em:

DE MORAIS, D. M.; SPOSTO, R. M. Propriedades Tecnológicas e Mineralógicas das Argilas e suas Influências na Qualidade de Blocos Cerâmicos de Vedação que Abastecem o Mercado do Distrito Federal.
Cerâmica Industrial, São Paulo, v. 11, n. 5/6, 2006, pp. 35-38, Setembro/Dezembro.

DURÃES, R. F. Como iniciar uma indústria de tijolos - parte I. Cerâmica, São Paulo, v. 29, 1983, p. 17-A.

MACEDO, R. S.; MENEZES, R. R.; NEVES, G. A.; FERREIRA, H. C. Influência de aditivos na produção de blocos cerâmicos. Cerâmica, São Paulo, v. 54, 2008, pp. 411-417. Disponível em: http: //dx.doi.org/10.1590/S0366-691320080003 00017. Acesso em:

MEDEIROS, R. R.; GONÇALVES, W. P.; CARTAXO, J. M.; FERREIRA, H. S.; NEVES, G. A.; FERREIRA, H. C. Influência do uso de resíduo de quartzito na expansão por umidade de massas de revestimentos cerâmicos planos. Cerâmica, São Paulo, v. 63, 2017. pp. 134-142.

Disponível em: http://dx.doi.org /10.1590/0366-69132017633662105.

Acesso em:

MOTTA, J. F. M.; ZANARDO, A.; CABRAL JÚNIOR, M. As Matérias-Primas Cerâmicas. Parte I: O Perfil das Principais Indústrias Cerâmicas e Seus Produtos.

Cerâmica Industrial, São Paulo, v. 6, n. 2, 2001, pp. 28-39, Março/Abril.

RAMOS, S. O.; MACEDO, R. S.; CARTAXO, J. M.; MENEZES, R. R.; NAVARRO, L. N. L.; neves, G. A. Caracterização de argilas usadas para cerâmica estrutural. Revista Eletrônica de Materiais e Processos, Campina Grande, v. 5.3, 2010, pp. 65-70.

RIBEIRO, M. J.; FERREIRA, A. A. L.; LABRINCHA, J. A. Aspectos

Fundamentais Sobre a Extrusão de Massas de Cerâmicas Vermelhas. Cerâmica Industrial, v. 8, n. 1, pp. 37-42, 2003, Janeiro/Fevereiro.

ROCHA BARRETO, I. A.; DA COSTA, M. L. Sintering of red ceramics from yellow 
Amazonian latosols incorporated with illitic and gibbsitic clay. Applied Clay Science, Netherlands, v. 152, 2018, pp. 124-130. Disponível em: https://doi.org/10.1016 /j.clay.2017.11.003. Acesso em:

SAMUDIO PÉREZA, C. A.; PADUANI, C.; ARDISSON, J. D.; GOBBI, D.; THOMÉ, A. Caracterização de Massas Cerâmicas Utilizadas na Indústria de Cerâmica Vermelha em São Domingos do Sul - RS. Cerâmica Industrial, São Paulo, v. 15 , n. 1,2010 , pp. 38 - 43, Janeiro/ Fevereiro.

SANCHEZ, E.; GARCÍA, J.; GINÉS, F.; NEGRE, F. Aspectos a Serem Melhorados nas Características e Homogeneidade de Argilas Vermelhas Empregadas na Fabricação de Placas Cerâmicas. Cerâmica Industrial, São Paulo, v. 1, n. 3, 1996, pp. 13 - 26, Julho/Agosto.

SANTOS, C. V. P.; SILVA, A. R.; GUIMARÃES FILHO, M. A. S.; FAGURY NETO, E.; RABELO, A. A. Índice de Plasticidade e Análise Racional de Argilas de Marabá (PA) para Avaliação das Zonas de Extrusão. Cerâmica Industrial, São Paulo, v. 17, n. 2, 2012, pp. 25-27. Disponível em: http://dx.doi.org/10.4322 /cerind.2014.009. Acesso em:

SOUZA, J. V.; SOUZA SANTOS, P. Ensaios preliminares de argilas visando a utilização cerâmica. Cerâmica, v. 10, n. 38, 1964, p. 2.
SOUZA SANTOS, Pérsio de. Ciência e Tecnologia de Argilas - v.1. 2. ed. São Paulo: Editora Edgard Blucher Ltda., 1989, p. 175-209.

TEIXEIRA, S. R.; DE SOUZA, DE SOUZA, S. A.; MOURA, C. A. I. Mineralogical characterization of clays used in the structural ceramic industry in west of S. Paulo State, Brazil. Cerâmica, São Paulo, v. 47, n. 304, 2001, pp. 204-207. Disponível em: http://dx.doi.org/10. 1590/S0366-69132001000400007. Acesso em:

VIEIRA, C. M. F.; FEITOSA, H. S.; MONTEIRO, S. N. Avaliação da Secagem de Cerâmica Vermelha Através da Curva de Bigot. Cerâmica Industrial, São Paulo, v. 8, n. 1, 2003, pp. 42-46, Janeiro/Fevereiro.

VIEIRA, C. M. F.; MONTEIRO, S. N.; DUAILIBI FILHO, J. Formulação de Massa de Revestimento Cerâmico com Argilas Plásticas de Campos dos Goytacazes (RJ) e Taguá (SP). Cerâmica Industrial, São Paulo, v. 6, n. 6, 2001, pp. 43-49, Novembro/Dezembro.

VIEIRA, C. M. F.; TERRONES, L. A.; SÁNCHEZ, R.; MONTEIRO, S. N. Características e efeito da fração granulométrica $<2 \mu \mathrm{m}$ no comportamento de queima de uma argila. Cerâmica, São Paulo, v. 53, 2007, pp. 249-254. Disponível em: http://dx.doi.org/10.1590/S036669132007000300006. Acesso em: 